\title{
DESIGN OF LOW POWER MEDICAL DEVICE
}

\author{
Wei Cai ${ }^{1}$ and Frank $\mathrm{Shi}^{2}$ \\ ${ }^{1}$ Department of Electrical Engineering and Computer Science, \\ University of California, Irvine, USA \\ ${ }^{2}$ HHS School of Engineering, University of California, Irvine, USA
}

\begin{abstract}
This paper describes the design of an MMIC phase shifter which can be used in a 4-channel 26-28 GHZ transmitter IC. The MMIC phase shifter is used for 5G RF front Ended applications. MMICs usually include power amplifiers with 4-bit digital phase shifters to make the phase adjustable. The whole design was used for the transmit chain of a mobile device and in a base-station.Future topology will continue to be improved.
\end{abstract}

\section{KEYWORDS}

Doherty Power Amplifier, MMIC, phase shifter

\section{INTRODUCTION}

5G technology is becoming a hot topic these days; many universities and companies are putting effort and money into this area. The 5G standard is not fixed yet, so all of the major players want to position themselves as key players in the market, since it can provide wide bandwidth, faster speed, and higher date rates. All the benefits which the current $4 \mathrm{G}$ network cannot provide. With the improved performance, a customer can download a high definition TV show in a second, or a high virtual reality gaming show; It will enable new markets, technologies and applications[1].

Since $5 \mathrm{G}$ is still under construction, the possible spectrum will be much higher than current cellular frequencies - possibly around $27 \mathrm{G}$, or $60 \mathrm{G}$. Because all the frequencies under $3 \mathrm{G} \mathrm{Hz}$ are taken, he higher spectrum must be used. Therefore, for the $5 \mathrm{G}$ radio frequency, higher frequency will be used, since it will also reduce the interference with currently used frequencies. Mm-waves are a good option in the USA and many Asian countries. Research is looking at using phased array of antennas to transmit power[2][3]. Due to the high frequency, the size is small which is good to save cost. Bands within $27 \mathrm{GHz}$ to $29.5 \mathrm{GHz}$ are also an excellent solution for $5 \mathrm{GHz}$ radio research.

The Figure 1 shows a $28 \mathrm{GHz}$ transmit IC RF front end circuit. It shows a 4 element antenna array, with each antenna controlled by a phase adjusted power amplifier. The phase shift cannot just change the phase but also reject the harmonics in the receive band[1].

DOI : $10.5121 /$ vlsic.2017.8201 
International Journal of VLSI design \& Communication Systems (VLSICS) Vol.8, No.2, April 2017

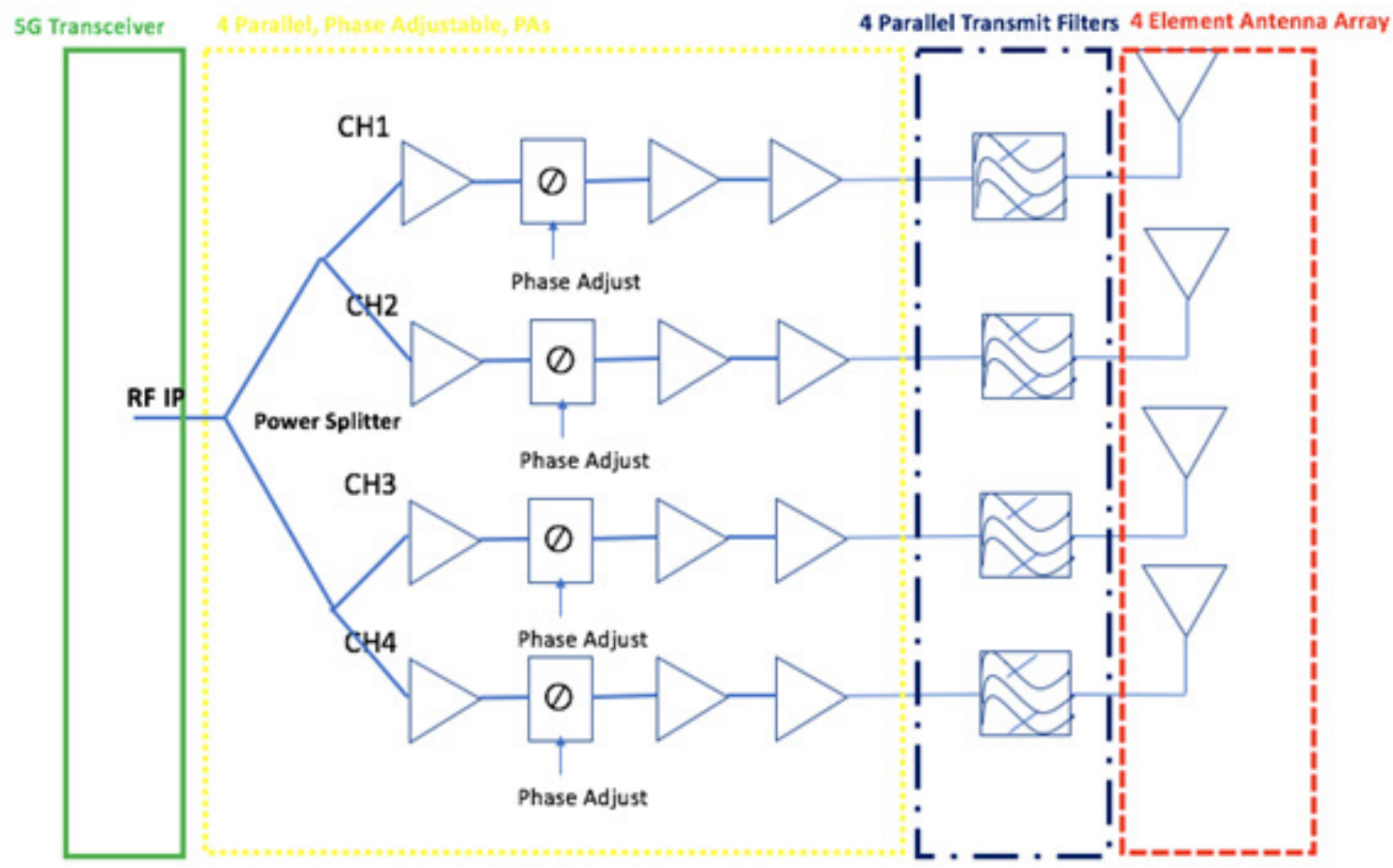

Figure1: MMIC schematic

\section{BACKGROUND}

For transmitters, power amplifiers (PA) are always a central element, which can highly affect the linearity and power efficiency of the whole system[4]. For a high efficiency PA at a wide range of output power the Doherty Power Amplifier (DPA) is a good solution, since it has excellent linearity while dealing with amplitude modulated signals. In this paper, a design for a low power and high linearity DPA for wireless networks is explored.

Table 1. DPA Comparasion.

\begin{tabular}{|l|l|l|l|l|}
\hline Type & Output Power & Efficiency & RF Band & Mod. Band \\
\hline Class AB & High & Low & High & High \\
\hline DPA & High & High & Low & High \\
\hline Outphasing & High & Medium & Low & Low \\
\hline Envelope tracking & Medium & High & High & Low \\
\hline Bias Modulation & Low & High & High & Low \\
\hline
\end{tabular}

Because of the demands of high data rates with specific modulation, high efficiency with an excellent peak to average power ratio is required[5]. Class AB PAs are well suited for the maximum output power, but their efficiency is low. The DPA is highly linear using digital predistortion, making it a suitable solution for $3 \mathrm{G}$ and $4 \mathrm{G}$ handset systems, as seen in Table 1 . The combination of a DPA and digital predistortion can achieve high average efficiency and the required linearity. 
International Journal of VLSI design \& Communication Systems (VLSICS) Vol.8, No.2, April 2017

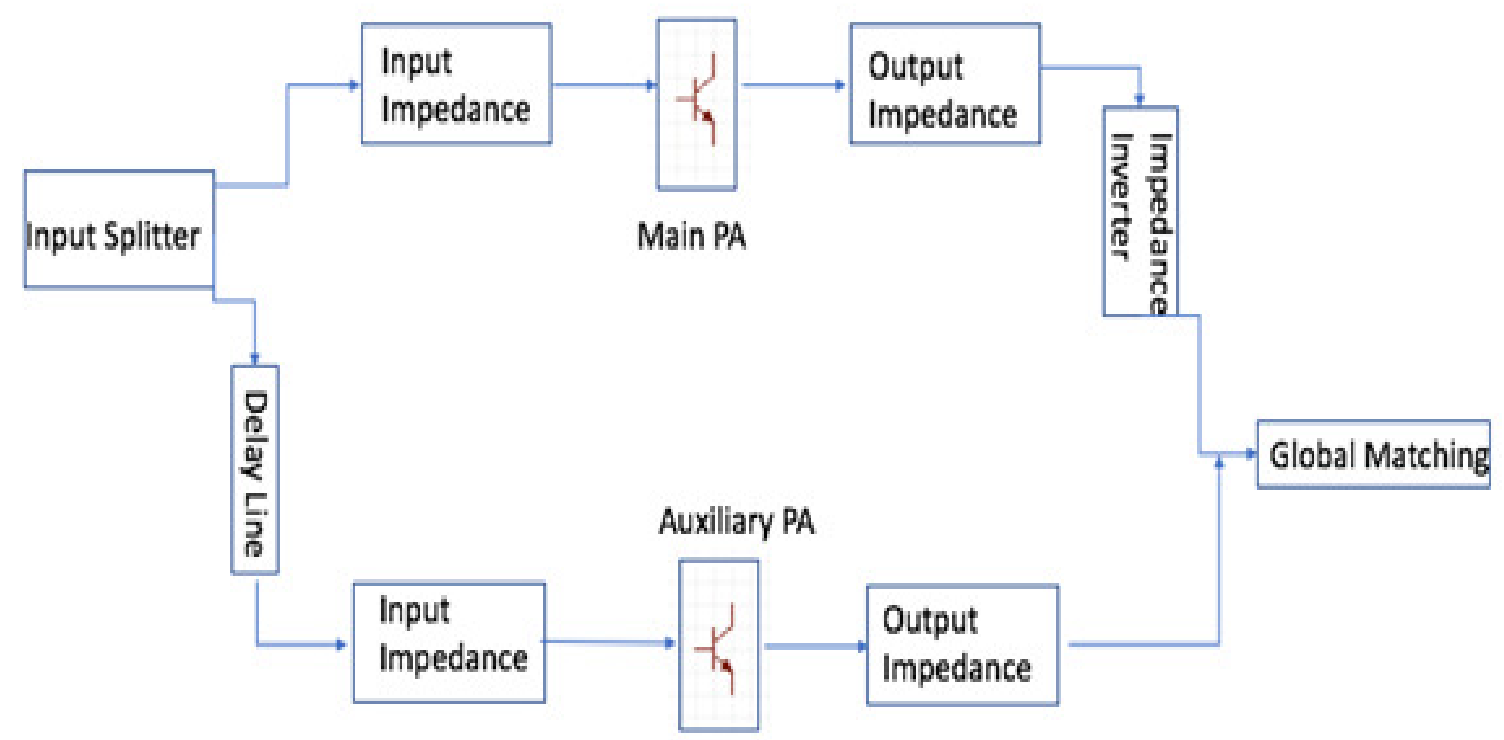

Figure2. DPA basic topology

The Doherty amplifier shown in Figure 2 has two PAs in parallel. The two PAs are both on when the amplifier is at full power. Only one PA is on when the power draw is low. For such a configuration, when the auxiliary PA is off and the total power draw is reduced, the load impedance of the main PA will be increased, allowing it to operate at higher efficiency. Such a topology would provide higher efficiency compared with a single power amplifier. Implementing quarter wavelength lengthscan be done by using lumped element impedance inverters.

\section{DESIGN}

Cadence RF specture was used to simulate a two stage DPA design. Each amplifier was simulated first at DC and S-parameters condition, to ensure functional operation. Then harmonic balance simulation was performed to ensure stability conditions. The last step was to combine the two amplifiers and the impedance inverters to form the base control DPA and perform a performance simulation. The input and output impedance inverters and the interstage match between Q1hp and $\mathrm{Q} 2_{\mathrm{hp}}$ were adjusted to improve the phase match between the two amplifiers of the DPA, to optimize power transfer[6]. A base control DPA was analyzed, designed and simulated.

The basic structure of the PA cell is shown in Figure 3[6]. The main PA, which is a carrier amplifier is the low power amplifier. The auxiliary PA, or the peaking amplifier will be referred to as the high-power amplifier.All the devices are connected in series, making the device stronger. The resistors are applied to the bias while also connecting to the base of each device, preventing current hogging. Resistors also improve the device's stability at all frequencies. Capacitors are used to couple with the RF input while also connecting to the base of each cell. Due to the feedback of gate to source this topology can make a stable device at all frequencies. 
International Journal of VLSI design \& Communication Systems (VLSICS) Vol.8, No.2, April 2017

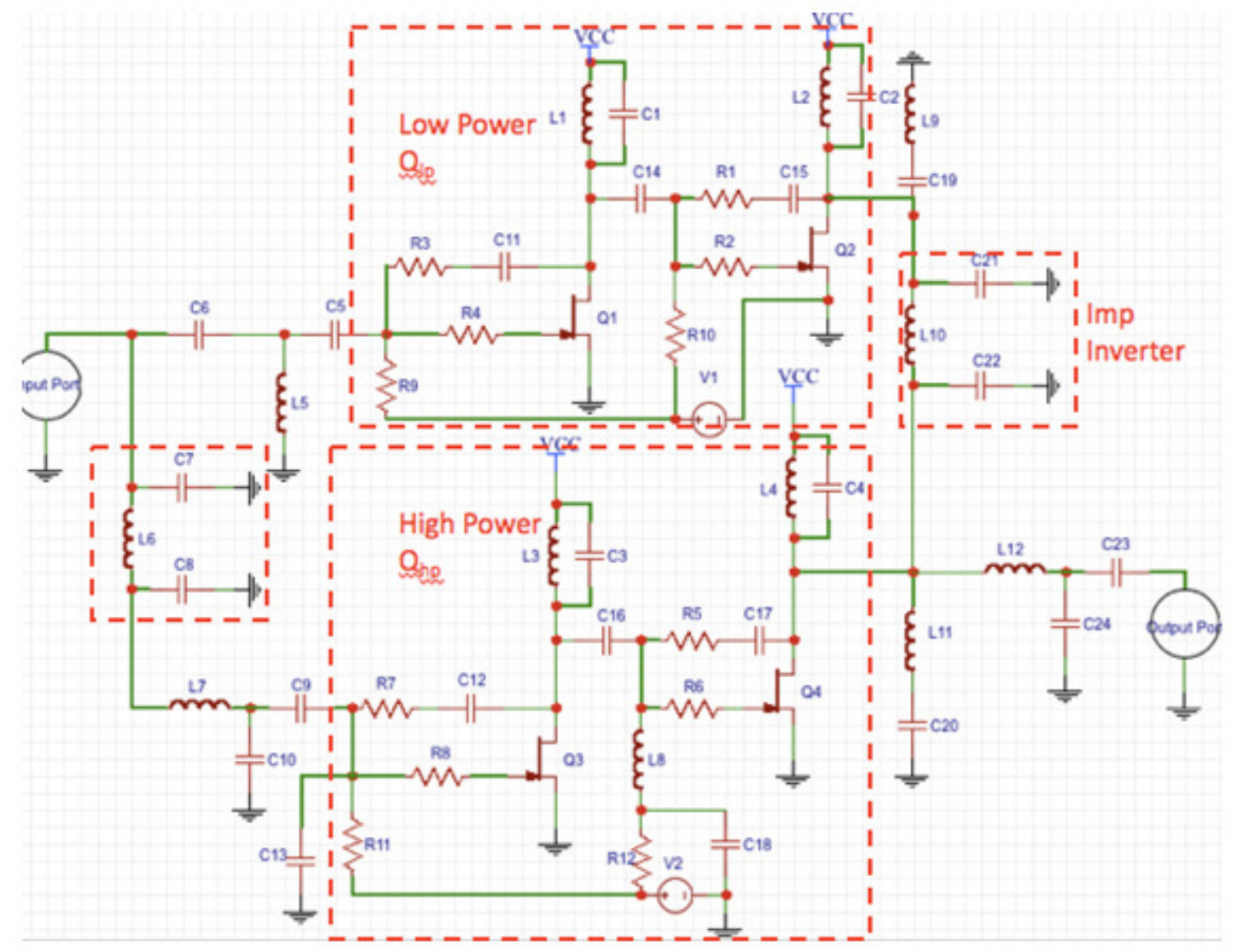

Figure 3. Whole DPA topology.

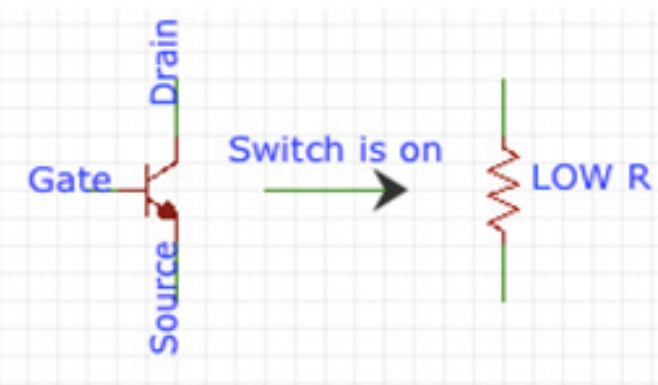

(a)

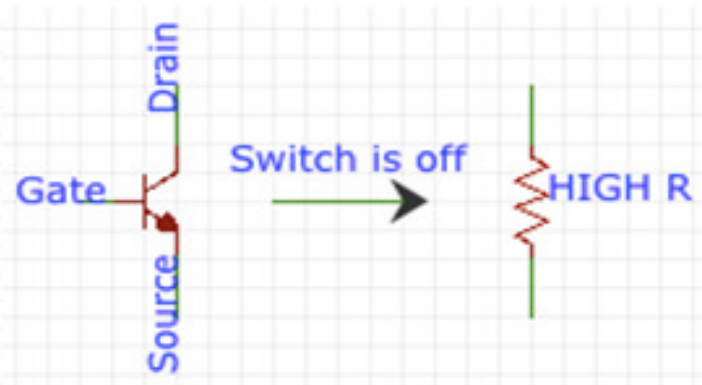

(b)

Figure 4: Switch MOSFET working condition (a) Switch on(b) Switch off

As seen in the Figure 4, when the voltage was forward-biased, the switch was on - this can be modeled as a small resistor. When the switch is off, the simplified circuit can be approximated as a large resistor. The phase shifter was designed as a high-pass/low-pass operating phase shifter with switching MESFETs. When a switching MESFET had a forward bias, it was modeled as a low resistor. When it had a reverse bias, it was modeled as a large resistor. 


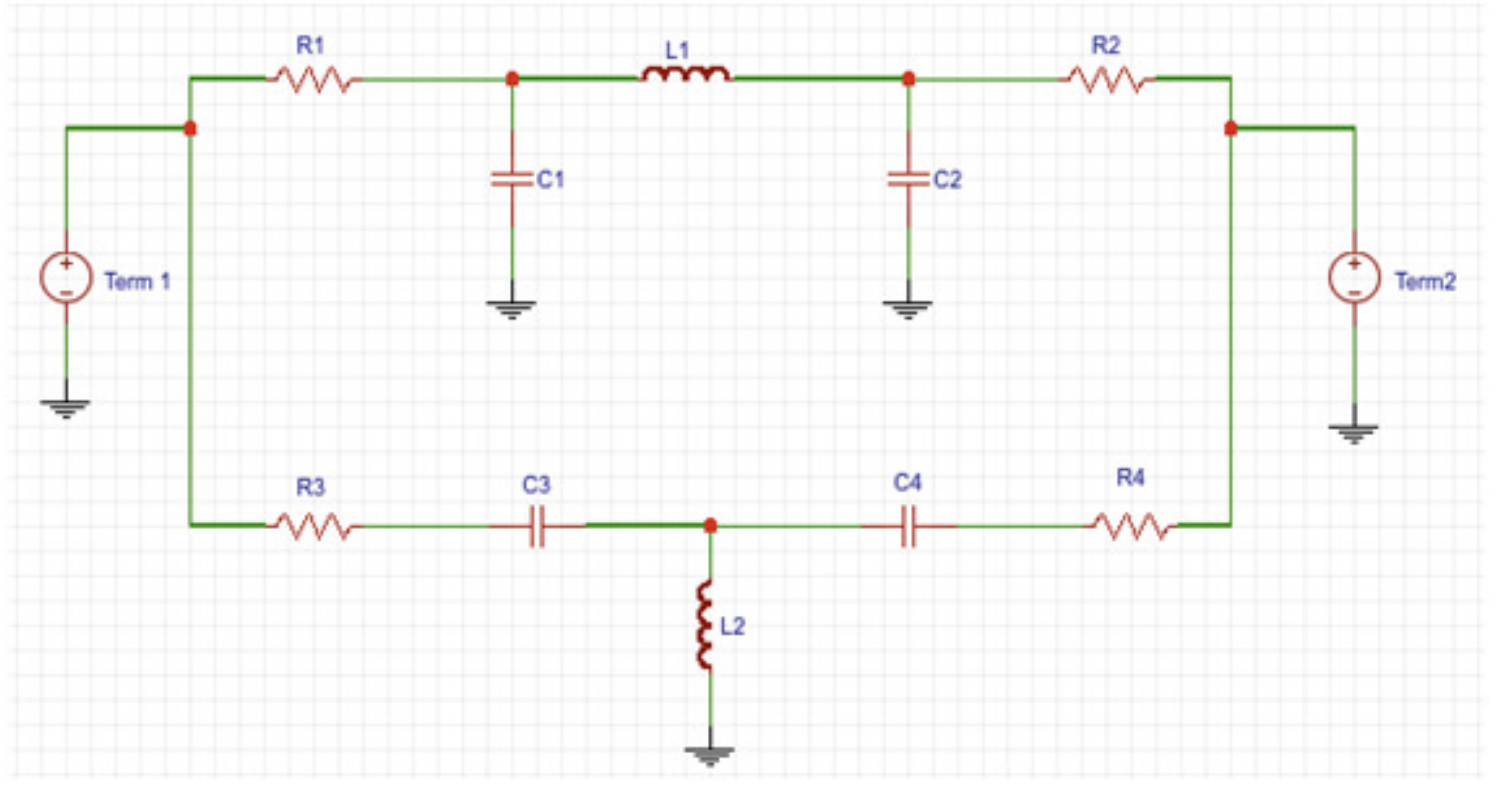

Figure 5: Phase shifter Schematic

The phase shifter as seen in Figure 5 is using a switched high-pass/low-pass filter topology[1]. High pass filters have better a phase advance compared to low pass filters because it is constant under relatively wide bandwidth. Optimization of the components seeks to get better performance, such as: phase difference with a minimum amplitude difference. The overall better solution is to get lower insertion loss as well as high isolation loss.

A high-performance phase shifter would have a steady amplitude response under some range of phase change. Though there would be always some change limiting the amplitude change leads to better performance. When the reflected waves changes, the phase shifter would also change, more detailed design simulation can be seen in Table 2 .

Table 2 Phase Shifter sizesfF

\begin{tabular}{|l|l|}
\hline Parameter & Size (Unit) \\
\hline R1, R2 & $20 \mathrm{ohm}$ \\
\hline R3, R4 & $0.05 \mathrm{ohm}$ \\
\hline L1,L2 & $0.683 \mathrm{nH}$ \\
\hline C1,C2,C3,C4 & $22.6 \mathrm{Ff}$ \\
\hline
\end{tabular}




\section{RESULTS}

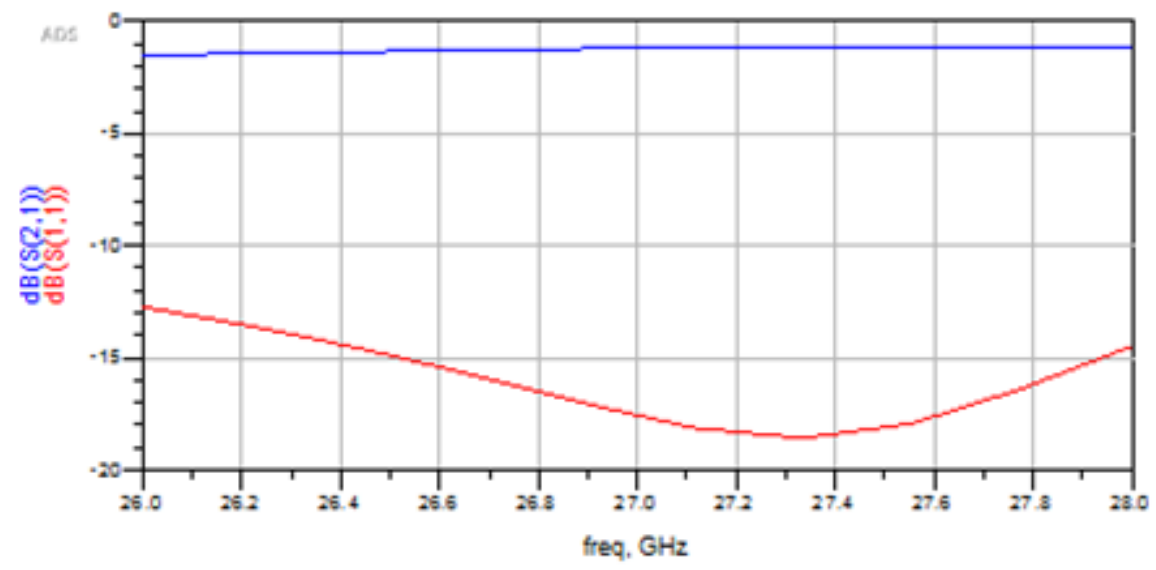

(a)

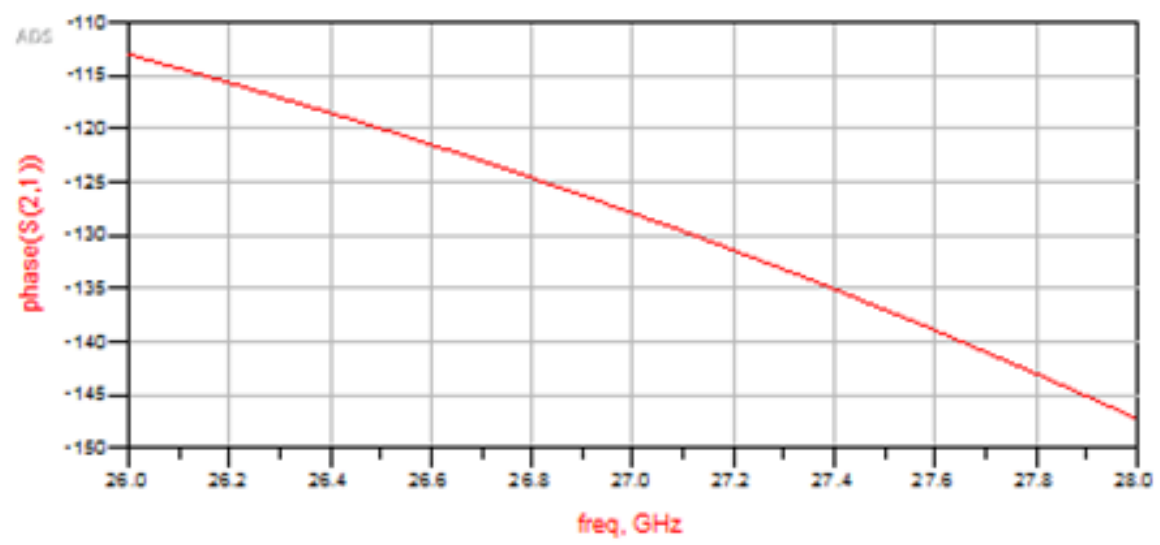

(b)

Figure 6. Phase Shifter (a) Magnitude (b) Phase

Figure 6 shows the results for the mmic phase shifter. The S11 and S21 mangitudes achieve -10 $\mathrm{dB}$ and $-1 \mathrm{~dB}$, respectively. The phase difference of the S21 could be -110 to -145 degrees between $26 \mathrm{GHz}$ to $28 \mathrm{GHz}$.

\section{CONCLUSIONS}

Simulation of an RF DPA using cadence software, based on IBM's SI 180nm technology process, is presented. Simulation of an RF phase shiftier using ADS software is also presented. This RF health care applications has been realized, but the current design still has room for further improvement for future requirements. To realize this, future improvement is needed. 
International Journal of VLSI design \& Communication Systems (VLSICS) Vol.8, No.2, April 2017

\section{REFERENCES}

[1] Stuart Glynn and Liam Devlin,"A 28GHz, 4-channel Phase Adjustable Power Amplifier IC for 5G front-ends",http://www.plextekrfi.com/wp-content/uploads/5g_4_channel_tx_ic.pdf

[2] Ma, Liangping, Wei Chen \&Dharm Veer, Gregory Sternberg, Weimin Liu, \&YuriyReznik, (2015) "Early Packet Loss Feedback for WebRTC-Based Mobile Video Telephony over Wi-Fi", In 2015 IEEE Global Communications Conference (GLOBECOM), pp. 1-6.

[3] Chen, Wei, Liangping Ma \&Chien-Chung Shen, (2015) "Congestion-aware MAC layer adaptation to improve video teleconferencing over wi-fi", Proceedings of the 6th ACM

[4] Wei Cai\&Frank Shi, (2016) "High Performance SOI RF Switch for Healthcare Application", International Journal of Enhanced Research in Science, Technology \& Engineering, Vol. 5, Iss. 10, pp23-28.

[5] Vittorio Camarchia, Marco Pirola, Roberto Quaglia, SeunghoonJee, Yunsung Cho, and Bumman Kim, (2015)“The Doherty Power Amplifier: Review of Recent Solutions and Trends",IEEE Trans on Microwave Theory and Tech, VOL. 63, NO. 2, pp 559-571.

[6] Darren W. Ferwalt, (2003) "A Base Control Doherty Power Amplifier Design for Improved Efficiency in GSM Handsets", MS thesis ,http://ir.library.oregonstate.edu/xmlui/bitstream/handle/1957/11722/Darren_W_Ferwalt.pdf

\section{AUTHORS}

Wei Cai is a graduate student at the University of California, Irvine, CA. She received her Masters degree from Dept. of Electrical Engineering, University of Hawaii at Manoa and Bachelor degree from Zhejiang University, China. Her research interests include device physics simulation, analog/ RF circuit design.

Frank Shi is an IEEE Fellow (named in 2011) and a recipient of the Sustained Outstanding Technical Achievement Award from the IEEE-CPMT (Component Packaging and Manufacturing Technology) Society, 2010. His specific interdisciplinary research activities focus on (1) Packaging \& Manufacturing Technologies for Optoelectronic Devices, (2) Materials for Optoelectronic Device Packaging \& Manufacturing, and (3) IC Packaging and Manufacturing issues. 